\title{
André Jolles, lecteur du Décaméron : récit-cadre, nouvelles et la question de l'unité
}

André Jolles lettore del Decameron: la «cornice», le novelle e la questione dell'unità

\section{Philippe Guérin}

\section{OpenEdition}

\section{Journals}

Édition électronique

URL : http://journals.openedition.org/cei/3125

DOI : 10.4000/cei.3125

ISSN : 2260-779X

\section{Éditeur}

UGA Éditions/Université Grenoble Alpes

Édition imprimée

Date de publication : 30 décembre 2016

Pagination : 85-108

ISBN : 978-2-84310-337-7

ISSN : $1770-9571$

\section{Référence électronique}

Philippe Guérin, «André Jolles, lecteur du Décaméron : récit-cadre, nouvelles et la question de l'unité », Cahiers d'études italiennes [En ligne], 23 | 2016, mis en ligne le 23 janvier 2017, consulté le 26 mars 2021. URL : http://journals.openedition.org/cei/3125 ; DOI : https://doi.org/10.4000/cei.3125 


\title{
ANDRÉ JOLLES, LECTEUR DU DÉCAMÉRON : RÉCIT-CADRE, NOUVELLES ET LA QUESTION DE L'UNITÉ
}

\author{
Philippe Guérin \\ Université Sorbonne Nouvelle - Paris 3, CERLIM \\ (Centre d'Études et de Recherche sur la Littérature Italienne Médiévale)
}

Sitôt qu'il veut représenter la réalité totale et multiple de la vie de son temps, Boccace renonce à l'unité de l'ensemble : il écrit un livre de nouvelles, dans lequel un grand nombre de sujets se côtoient, rassemblés seulement par l'intention générale de fournir un divertissement de qualité.

On aura reconnu le jugement d'Auerbach, familier à nombre de boccaciens, énoncé dans le chapitre de Mimésis consacré à frate Alberto ${ }^{\mathrm{I}}$. Par-delà les inexactitudes et les points contestables ${ }^{2}$, l'analyse de la nouvelle IV 2 reste à bien des égards exemplaire, pour sa façon de montrer comment par les moyens du style (du travail du style), Boccace «embrasse du regard et dépeint de la manière la plus concrète toutes les catégories sociales, les professions et les conditions de son temps», pour "atteindre [grâce au précédent de Dante] le point à partir duquel il est possible d'embrasser du regard [de nouveau] le monde contemporain tout entier et ses phénomènes, de saisir ceux-ci dans leur multiplicité et de les reproduire dans une langue expressive et docile ${ }^{3}$.

Mais le jugement d'ensemble portant sur le Décaméron par le philologue allemand ne va pas de soi. La question fondamentale qui est posée par notre citation inaugurale (sous-jacente du reste à la comparaison

I. E. Auerbach, Mimésis. La représentation de la réalité dans la littérature occidentale, traduit de l'allemand par C. Heim, Paris, Gallimard, I968, p. 238. L'ouvrage, rappelons-le, paraît en 1946 [Dargestellte Wirklichkeit in der abendländischen Literatur, Berne, Munich, Francke].

2. On ne peut pas dire qu'il n'y a chez Boccace ni gravité ni sublime (ibid., p. 227; que l'on pense par exemple aux nouvelles IV I, ou X 8); il nous paraît faux de dire que la nouvelle de Ciappelletto (I I) «n'a pas d'autre fin que d'amener deux scènes bouffonnes" et que «Boccace n'a raconté cette scandaleuse histoire qu'en vue de l'effet comique» (ibid., p. 239), etc. Voir aussi A. Casadei, Per Auerbach contro Auerbach, dans Id., Letteratura e controvalori. Critica e scritture nell'era del web, Rome, Donzelli, 20I4, p. 3-37.

3. E. Auerbach, Mimésis, ouvr. cité, p. 222, 229. 
menée avec Dante, au bénéfice évidemment de ce dernier) est bien celle de l'unité, ici niée - et il est frappant à cet égard de constater que nulle part dans le chapitre en question, ce qui apparaît dès l'abord au lecteur du Décaméron comme un puissant facteur de cohésion au moins structurelle, à savoir la présence d'un récit-cadre aux proportions imposantes et aux caractéristiques largement inédites, cette "cornice», comme l'on dit le plus souvent en italien, n'est mentionnée - sinon indirectement par l'allusion au divertissement destiné à la société raffinée des membres de la «lieta brigata» (mais pas même désignée comme telle) ${ }^{4}$.

Écoutons à présent André Jolles. Il s'agit des lignes qui ouvrent l'étude de 1923 (vingt-trois ans, donc, avant Mimésis) consacrée au Décaméron de Boccaces. Banc d'essai, approche empirique de ce qui, décanté, devait donner la construction théorique des «formes simples» telles que nous les connaissons depuis l'édition de 1930? Assurément ${ }^{6}$. Mais je propose pour l'instant de lire ce chapitre de manière autonome. Voici donc (en italien, parce que, sauf erreur, il n'y a pas de ce texte de traduction française, et que le néerlandais est inconnu non seulement de l'auteur de ces lignes, mais aussi de la quasi-totalité, sans doute, de ses lecteurs potentiels) :

[...] è davvero possibile rinvenire un filo rosso che unisce questi cento racconti diversi?

Alla domanda si può senza dubbio rispondere affermativamente, aggiungendo che solo colui che s'impegna nella ricerca di tale filo potrà comprendere il Decameron per intero, e solo chi lo abbia trovato sarà nelle condizioni di esprimere un giudizio sul valore dell'opera e il significato morale del libro' ${ }^{7}$.

4. Ibid., p. 225 : «La narration ne se propose plus de fournir un exemplum moral, elle ne se propose pas non plus de satisfaire simplement le besoin de rire des gens du peuple : elle ambitionne de divertir un cercle de jeunes hommes et de jeunes femmes de condition supérieure, instruits, qui prennent plaisir aux jeux de la vie des sens, qui possèdent de la sensibilité, du goût et du jugement; c'est pour que ses contes remplissent cette fonction que Boccace a créé le cadre où il les situe. " Il est vrai que dans Zur Technik der Frührenaissancenovelle in Italien und Frankreich (traduction italienne La tecnica di composizione della novella, Rome-Naples, Theoria, 1984, p. 25, cité par S. Contarini dans A. Jolles, I travestimenti della letteratura. Saggi critici e teorici (I897-1932), S. Contarini (éd.), premessa di E. Raimondi, Milan, Bruno Mondadori, 2003, p. XLIII), Auerbach souligne l'effet de contraste entre description de la peste de l'Introduction à la première journée du Décaméron et le monde léger de la «lieta brigata». V. Branca, grand ordonnateur des études boccaciennes dans la deuxième moitié du $\mathrm{xx}^{\mathrm{e}}$ siècle, soulignera lui aussi cette fonction de mise en relief assignée à la "cornice» : voir Boccaccio medievale, Quarta edizione accresciuta, Florence, Sansoni, 1975 (1956), p. I8 ("Ma per dare più chiarezza e più saldezza a questo disegno [l'itinéraire du Décaméron modelé sur la structure de la "comédie» au sens médiéval du terme], a questo svolgimento ideale, il Boccaccio lo racchiude in una "cornice" che ne sottolinei in qualche modo i momenti salienti $[\ldots] »)$.

5. Voir A. Jolles, Il Decameron di Boccaccio, dans I travestimenti della letteratura, ouvr. cité, p. 57-II6. Il s'agit de la reprise en néerlandais, avec l'aide de J. Huizinga, de la préface de la traduction allemande de I92I du Décaméron (voir la Nota ai testi de S. Contarini, ibid., p. LVII).

6. Voir notamment ibid., les p. 58-59, sur lesquelles nous reviendrons.

7. Voir ibid., p. 57 (les passages soulignés le sont par nous). 
C'est même là ce qui paradoxalement rend la lecture intégrale — continue et progressive — non absolument nécessaire pour saisir le sens de l'œuvre, comme le précisent les tout premiers mots de l'essai en question. Pourvu, on le verra, que l'on ait identifié la clé d'accès.

Mais Jolles d'enchaîner :

Da questo punto di vista l'introduzione premessa alle novelle delle dieci giornate costituisce un indizio probatorio che rivela il posto occupato dal libro intero nella letteratura e nella vita dello scrittore, il primo tentativo di indurre il lettore a considerare questopera come un'unità e a leggerla come un insiemes.

Ce n'est donc pas au problème de la nouvelle dans ses rapports avec le tragique (et aux divergences de vue entre nos deux critiques et historiens de la littérature à ce sujet aussi), point excellemment évoqué naguère par Silvia Contarini ${ }^{9}$, que je vais directement m'attacher, ni d'abord à la «forme simple» ou au "geste verbal», voire à la "disposition mentale» qui sont au point de départ des «formes actualisées» aboutissant à la "forme artistique» (ou "savante $»^{\mathrm{I}}$ ) ayant pour nom «nouvelle» — autrement dit à la question toujours débattue du genre ${ }^{\text {II }}$; mais au rapport entre récitcadre et groupement de nouvelles, à ses conséquences sur l'appréhension du système de représentation du monde qui se joue dans le Décaméron et, in ultimo, à la question de la morale, pointée comme on l'a vu plus haut ab initio (Auerbach refusant pour sa part qu'il y ait une morale du Décaméron $\left.{ }^{12}\right)$. Les intuitions de Jolles me semblent à cet égard d'un très grand intérêt.

8. Ibid. (et toujours souligné par nous).

9. Voir S. Contarini, "Forme artistiche”: Jolles e la teoria della novella», dans G. M. Anselmi et al., (éd.), Boccaccio e $i$ suoi lettori. Una lunga ricezione, Bologne, Il Mulino, 20I3, p. I09-I27, ici p. I25-I27.

Io. "Formes savantes, cela veut dire pour nous des formes littéraires qui sont précisément conditionnées par les choix et par les interventions d'un individu, formes qui présupposent une ultime et définitive fixation dans le langage, formes qui ne sont plus le lieu où quelque chose se cristallise et se crée dans le langage, mais le lieu où la cohésion interne la plus haute est atteinte dans une activité artistique non répétable.» (A. Jolles, Formes simples, traduit de l'allemand par A. M. Buguet, Paris, Seuil, «Poétique», 1972, p. I44.)

II. Voir par exemple H. R. Jauss citant Jolles dans Littérature médiévale et théorie des genres, dans G. Genette, H. R. Jauss, J.-M. Schaeffer, R. Scholes, W. D. Stempel, K. Viëtor, Théories des genres, Paris, Seuil, I986, p. 37-76. Voir aussi M. Picone, Boccaccio e la codificazione della novella. Letture del Decameron, N. Codrey, C. Genswein, R. Pittorino (éd.), Ravenne, Longo, 2008 ; ainsi que les essais sur la question des volumes collectifs suivants : M. Picone, G. Di Stefano, P. D. Stewart (éd.), dans La Nouvelle. Genèse, codification et rayonnement d'un genre médiéval, actes du colloque international de Montréal (McGill University, I4-I6 octobre 1982), Montréal, Plato Academic Press, 1983; La novella italiana, Atti del Convegno di Caprarola (19-24 settembre 1988), Rome, Salerno, I989, 2 vol. (voir plus particulièrement les contributions d'E. Malato, C. Segre, M. Picone, A. Vàrvaro).

I2. Voir E. Auerbach, Mimésis, ouvr. cité, p. 236-237, où le romaniste allemand prétend que la «morale de l'amour » de Boccace ne présente aucun caractère sérieux; et même s'il avait affirmé (ibid., p. 230) qu’à la "vision synthétique du monde» qui est celle de Boccace, «s'associe une conscience critique, ferme encore qu'élastique 
«Introduction", dit Jolles (en tout cas dans la traduction dont nous disposons). Comme nous l'avons rappelé, "cornice», en italien, malgré le risque de méprises dont il est gros ${ }^{13}$. Précisément parce que la question du lien organique qui relie avec force ledit "récit-cadre", voire "récit contenant" ou "récit porteur" (comme je crois qu'il serait préférable de le désigner) à l'ensemble des récits du niveau principal (les nouvelles), n’a plus guère de pertinence si l'on considère ce métarécit comme un simple "cadre" à visée essentiellement ornementale (un ornement gothique flamboyant de polyptyque, pour qui tente, avec de bonnes raisons, mais dont les conséquences induites peuvent à leur tour être cause de fourvoiement, de "médiévaliser" Boccace). Un élément structurel qui, ayant une fonction purement architecturale, resterait extrinsèque à la matière même des nouvelles $^{\mathrm{I}}$. Et que distinguerait au mieux, pour une critique récente avertie en

dans ses perspectives, qui, sans moraliser abstraitement, attribue aux événements leur valeur morale particulière avec toutes les nuances convenables"; et que l'«ironie" qui est la marque de l'auteur "présuppose un système complexe et multiple d'évaluations possibles [...]", c'est de fois en fois, au niveau de chaque nouvelle prise séparément, par le biais notamment de l'enregistrement des réactions et attitudes des personnages, que se modulent de tels jugements.

Sur l'éventuelle morale d'ensemble que l'on pourrait tirer du Décaméron, il semble bien que l'on soit encore majoritairement sur la ligne inaugurée par F. De Sanctis, pour qui la "comédie humaine» qui constitue la matière des cent nouvelles, ne dépasse pas le plan du divertissement ("qui il riso è per il riso, per passare malinconia, per cacciare la noia») et le jugement global de De Sanctis est un jugement de superficialité sur une œuvre d'où la dimension morale est au bout du compte absente (Storia della letteratura italiana [I870], les paragraphes 3, "Il mondo naturale e il mondo mitologico », et 4, "Il motivo comico e la sua origine intellettuale», du chap. IX consacré précisément au Décaméron; nous citons d'après F. De Sanctis, Storia della letteratura italiana, a cura di L. Russo, 2 vol., Milan, Feltrinelli, I956, I, p. 324 et p. 333).

13. Voir par exemple A. Asor Rosa, Decameron di Giovanni Boccaccio, dans Letteratura italiana. Le opere, vol. I, Dalle origini al Cinquecento, Turin, Einaudi, I992, p. 473-59I, ici p. 492 : "è il concetto stesso di "cornice" a dover esser rimesso in discussione, anche se continuiamo ad usarlo per la particolare sinteticità del termine. "Cornice", infatti, dovrebbe essere un puro inquadramento, un abbellimento esteriore, un bell'arabesco formalistico rispondente ad una logica di simmetrie molto medievale [...]». Notons qu'Asor Rosa lui aussi avait précédemment souligné (ibid., p. 475) la fonction du contraste entre évocation de la gigantesque catastrophe initiale et le reste de l'œuvre, sa logique "ordinatrice e umanamente razionale», le gigantesque acte créateur qui la produit.

I4. V. Branca, dans Boccaccio medievale, ouvr. cité, p. I4, évoque la "generale architettura gotica del Decameron: che rivela il suo grandioso disegno in maniera più appariscente nell'organismo ideale dell'opera e nella coerente "cornice" in cui l'autore ha voluto racchiuderlo». Et un peu plus loin (ibid., p. I8-I9) : «E questa "cornice", se è quasi uno schema poetico obbligatorio per la fantasia del Boccaccio, è caratteristico della letteratura e della retorica medievale e quasi inconcepibile fuori di essa.» Pour évoquer aussitôt après "il suo preminente valore decorativo". Mais Branca lui-même (ibid., p. 32), s'attachant plus particulièrement à l'Introduction à la première journée et plus précisément encore à la description de la peste, écrit : «La stessa comoda formula (vero "idolo" critico!) della "cornice", la quale avrebbe rappresentato l'unico meccanico legame fra le diverse narrazioni, contribuiva a isolare e a respingere, come una conferma senza appello, l'Introduzione in quella funzione esornativa e meramente esteriore». (Voir aussi L. Battaglia Ricci, Boccaccio, Rome, Salerno, 2000, p. 156, à propos de la «storia portante del libro — [...] quella che solo per convenzione si continua a indicare col termine evidentemente improprio di "cornice" "). Considération qu'il faut à mon sens étendre à l'ensemble du récit porteur — ce qui avait été entrevu par Branca dans son style à la fois fleuri et allusif ("gothique"), sans que les conséquences en soient véritablement tirées, V. Branca, Boccaccio medievale, ouvr. cité, p. 20 : «Ma anche su un altro piano, più intimo all'opera, più connesso alle sue stesse basi ideali, la 
matière de dispositifs sémiotiques, à travers la fonction de mise en valeur dévolue ordinairement au cadre, une visée métalittéraire de promotion du genre-nouvelle que Boccace est en train sinon d'inventer, du moins de codifier (si l'on veut recourir à un terme qui n'est pas lui non plus dépourvu d'ambiguïté) ${ }^{\mathrm{IS}}$, à quoi en tout cas il donne forme à travers une "pratique théorique" scandée d'intermèdes métadiscursifs, auctoriaux, ou bien internes à l'ordre diégétique.

Tâchons alors de voir ce qui fait du «racconto a cornice» [kadervertelling] " une "forme littéraire», ce qui l'élève à la dignité d'une "forme artistique», par nature composite - et destinée en tant que telle, selon Jolles (Jolles "morphologue», théoricien des "formes", précisément), à acquérir son indépendance vis-à-vis de son créateur, pour aussitôt vivre sa vie propre en se déployant en toute autonomie comme organisme autosuffisant, sous le signe de "l'objectivité du procès de développement des formes", procès irréversible d'où l'homme finit par s'effacer presque complètement ${ }^{16}$. Nous aurons en vue dans les paragraphes qui suivent, non pas tant la genèse d'une telle forme, les sources possibles de Boccace et l'inscription de son texte dans une typologie ${ }^{17}$, ni la "vie de la forme» en tant que telle, mais le principe d'articulation entre les deux niveaux (principaux) de la diégèse (ce sur-récit et les nouvelles racontées par les personnages dudit récit premier $)^{18}$. Parce que c'est la préoccupation de Jolles lui-même.

"cornice" rivela chiaramente l'architettura medievale del Decameron: quando cioè — adempiendo ad una delle sue funzioni precipue - svela le grandi linee della concezione del Decameron, dichiara teoreticamente le ragioni e gli ideali che regolano quelle diversissime e splendide rappresentazioni». (On n'en saura guère plus, sauf l'erreur — à notre avis — relative à l'interprétation de la Fortune; la fin de la phrase est soulignée par moi.)

Pour le maintien toutefois de "cornice», au nom du respect dû à ce que l'histoire de la réception a codifié (par commodité, donc, aussi), voir M. Picone, Il Decameron come macrotesto: il problema della cornice, dans M. Picone, M. Mesirca (éd.), Introduzione al Decameron, Florence, Cesati, 2004, p. 9-33, ici p. I3.

I5. Sur la question solidaire du "livre de nouvelles", voir L. Battaglia Ricci, Scrivere un libro di novelle. Giovanni Boccaccio autore, lettore, editore, Ravenne, Longo, 2013.

I6. Voir A. Jolles, I travestimenti della letteratura, ouvr. cité, p. 58.

I7. Sur cette question, les meilleures mises au point sont, je crois, celles de M. Picone : voir par exemple Il Decameron come macrotesto, art. cité, dont c'est précisément l'objet. Mais voir aussi n. II ci-dessus.

I8. Sur la question de l'unité globale de l'organisme Décaméron et, tout particulièrement, de son «intima virtù formativa" (p. 9), voir les pages de G. Getto intitulées La cornice e le componenti espressive del Decameron, dans Id., Vita di forme e forme di vita nel Decameron, [1957] 1972, terza edizione riveduta, Turin, Petrini, I972, p. I-33 (et voir l'utile rappel de la position de Vittorio Cian sur le sujet, ainsi que de ceux qui l'ont suivi, ibid., p. I6-I8). Getto met en avant lui aussi les raisons esthétiques du "contraste" entre description de la peste et atmosphère présidant au «novellare" (ibid., p. Io, I6 et passim; avec "funzione chiaroscurale», ibid., p. 30). En outre, et surtout, emboitant en quelque sorte le pas à De Sanctis, il situe cette "virtù formativa» sur un autre plan que celui de la morale stricto sensu (ibid., p. I4 et suiv.), en en définissant d'une formule un peu mystérieuse le sens comme «lirico-umano", et plus clairement comme un "saper vivere", o "arte di vita» (ibid., p. I6, conclusion p. 33). Voir également depuis l'importante contribution de Giorgio Bàrberi Squarotti, La "cornice» 
Mais qu'est-ce qu'une «forme» (et toute forme en général) ? Citons de nouveau le critique néerlandais pour une définition :

Ciò che chiamiamo forma è sempre una vittoria. Trasformando i suoni naturali in parole, i pensieri in frasi, le parole in versi dotati di metro e di ritmo, riportiamo sempre una sorta di vittoria sul caos, una creazione simile a quella della natura ${ }^{19}$.

Cela posé, après avoir passé en revue une première fois rapidement les différentes possibilités d'agrégation d'un ensemble de nouvelles, et esquissé une typologie sommaire (bipartie) des formes historiques de récits cadre, Jolles dégage leur point commun : «Ogni cornice tende [...] a proporre una lezione di vita o a enunciare una morale ${ }^{20}$." Il n'est guère besoin ici d'illustrer le propos concernant les règles de vie et la ligne de conduite que se donne la «lieta brigata», bien connues et toujours commentées d'abondance - victoire au moins momentanée contre le chaos. En revanche cette «morale» victorieuse instaure-t-elle quelque lien avec l'univers représentationnel des nouvelles et avec (au sens le plus neutre du mot) l'éthique, implicite ou non, qui s'en dégage, pour tracer quelque chose comme une signification morale d'ensemble du livre (pour reprendre les termes de notre première citation de Jolles)?

La réponse semble suggérée par une sorte de glissement logique au milieu du raisonnement sur le "racconto a cornice», quand l'attention, focalisée sur la "cornice», précisément (sur le récit en quoi elle consiste concrètement, croit-on comprendre), est dirigée vers l'ensemble constitué par les multiples pièces dont le montage aboutit au livre en sa totalité.

Il racconto a cornice ricerca gli esempi più diversi e lontani tra loro per enunciare una forma di saggezza quotidiana attraverso le situazioni più dissimili oppure per mettere l'accento su di una morale sostenuta con forza o rifiutata. Le espressioni «morale» e «saggezza quotidiana» non vanno intese in senso stretto, riferite alla filosofia, all'etica $\mathrm{o}$ alla morale, così come vengono intese dagli accademici e predicate dai pulpiti ${ }^{21}$.

Nous sommes entrés ce faisant dans le "monde de l'œuvre» (pour reprendre l'expression du Ricœur de La Métaphore vive) au niveau des

del Decameron o il mito di Robinson, dans Id., Il potere della parola. Studi sul Decameron, Naples, Federico \& Ardia, 1983, p. 5-63.

19. A. Jolles, I travestimenti della letteratura, ouvr. cité, p. 57-58 (souligné par moi). On remarquera que l'énoncé de Jolles n'est pas dépourvu d'ambiguïté, voire d'une possible contradiction interne : la transformation des sons naturels en mots est une victoire sur le chaos, mais c'est déjà ce que fait la nature elle-même (vaincre le chaos) lorsqu'elle crée...

20. Ibid., p. 6o. Et p. 6I, au terme du raisonnement sur ce point : «In conclusione, il racconto a cornice è una forma letteraria che si prefigge lo scopo di illustrare attraverso le immagini una morale o una filosofia di vita" (souligné par moi).

2I. Ibid., p. 60. 
récits singuliers eux-mêmes. Où se déploierait donc, en dehors de tout maniérisme comme, surtout, de tout allégorisme ${ }^{22}$, ce que nous définirions volontiers comme une "pensée de l'œuvre (littéraire)" (génitif subjectif), grosse d'une "philosophie" implicite. Et en tout cas, l'intention de Jolles est bien, insistons-y, de montrer que le Décaméron tout entier est un «livre moral $»^{23}$. Chez lui aussi, est soulignée la dialectique de la vie et de la mort qui, dans l'espace textuel du recueil, se résout, à l'intérieur de la parenthèse des quinze jours hors de Florence en proie à la peste, en jouissance de la beauté et en plaisir de la narration, mais au profit d'un utile qui va bien au-delà de la simple et ô combien incertaine conservation de soi ${ }^{24}$.

Après avoir examiné les rapports avec Dante, Jolles va donc consacrer un espace conséquent à ladite "cornice» du Décaméron, considérée dès lors en elle-même, en traquant d'abord les signes de l'autoportrait et en cédant au démon du biographisme ${ }^{25}$. Pour nous ici, toutefois, cette "cornice» est surtout le lieu où se conjuguent subtilement homogénéité et variété (tout d'abord ténue), dans une unité de style (allant de pair avec les personnages de la «brigata» semblables entre eux, et avec les journées dans les deux "villes» toujours organisées selon le même schéma), une unité qui cependant n'est pas monotonie : c'est le règne du détail parfois infime, mais porteur de diversité subtile, de distinctions d'abord menues qui se font de plus en plus nettes ${ }^{26}$. Cette dynamique discrète sans aucun doute signifie. Tout cela étant parfaitement fonctionnel au but recherché, consistant in fine à comprendre (au sens fort) en un tout unifié un monde hétéroclite - celui des nouvelles - dont il faut «tempérer la représentation ${ }^{27}$ ». Tempérer? Qu'est-ce à dire? Jolles se sert de la comparaison avec Chaucer (qui procède à l'inverse) pour montrer comment, même si le récit-cadre

22. Tel est (ibid., p. 88-93) le cœur du long développement consacré à Dante, se terminant par la mise en évidence de la voie originale qu'après des tâtonnements initiaux visant à l'imitation du maître, Boccace devait finalement trouver et emprunter : "Se non possiamo affermare che la Divina commedia e il Decameron parlano la stessa lingua, è indubbio che la prosa di Boccaccio possiede la stessa immediatezza ed evidenza della poesia dantesca, e che l'una ha origine dall'altra» (p. 93; Auerbach aussi ne cesse d'insister sur ce point, d'un Boccace entièrement redevable à Dante des possibilités expressives ouvertes par son maître déclaré).

23. Ibid., p. 65 .

24. Sur cette question vue sous l'angle du comique, des vertus thérapeutiques du rire, voir l'importante contribution d'E. Fenzi, «Ridere e lietamente morire. Un'interpretazione del Decameron », Per leggere, I2, 2007, p. I2I-I5O.

25. A. Jolles, I travestimenti della letteratura, ouvr. cité, p. 93-IoI.

26. Ibid., p. 97-98.

27. Ibid., p. 98: «La ragione per la quale Boccaccio ha voluto così è facile da intuire. La cornice deve, senza perdere il suo carattere di opera d'arte mobile, contenere come un tutto omogeneo novelle molto diverse e tra loro contrastanti; e come una ghirlanda regolare di foglie verdi, ornata solo di alcuni fiori colorati, deve temperare la rappresentazione viva e realistica delle diverse novelle.» 
doit être perçu comme une «histoire vraie», il est repoussé sur les bords pour permettre de représenter au plus près la réalité brute et crue des nouvelles, dont même les plus obscènes sont rendues acceptables sur un tel fond ${ }^{28}$. Question d'équilibre, aussi, entre une stylisation poussée qui, baignée d'une lumière raffinée faisant penser à celle filtrée par des vitraux, n'apparaît pas pour autant artificielle ou forcée, et, d'autre part, le plein jour, solaire et aveuglant, lui, du monde des nouvelles. La subjectivité étant du côté du narrateur pleinement «réaliste» de ces dernières, l'objectivité dans la description de l'environnement où elles surgissent ${ }^{29}$. Ou encore mémoire contre choses vues, voix opposées au style (et au ton de voix unique des personnages du récit-cadre $)^{30} \ldots$ Ou encore, pour l'historien des formes littéraires, filon provençal dans la «cornice» et filon épique du nord de la France dans les nouvelles (intéressante, cette intuition selon laquelle les nouvelles décaméroniennes auraient quelque chose à voir avec l'épopée) ${ }^{31}$. Mais avec le souci que la distance entre les deux régimes discursifs ne soit pas infranchissable, afin que se module le passage de l'un à l'autre (Dioneo étant la figure incarnée d'une telle médiation - quand ce n'est pas, à l'intérieur même du récit-cadre, la cornemuse de Tindaro accompagnant la danse des jeunes gens) ${ }^{32}$.

Jolles va pouvoir passer alors à la dernière section de l'essai, consacrée aux nouvelles elles-mêmes. Qu'y apprend-on qui puisse nous intéresser plus particulièrement ici? Une définition, d'abord :

Per novella intendiamo la rappresentazione di un fatto o di un avvenimento di significato chiaro e intenso, che suscita nel lettore un'impressione di verità. Tale avvenimento viene tradotto dalla novella in una forma che privilegia la rappresentazione dell'evento sui personaggi coinvolti. Ciò che importa è il fatto in sé [... $]^{33}$.

28. Ibid. Voir aussi le paragraphe concernant la Valle delle donne (ibid., p. Ioo) et la mise à distance en une énonciation de second degré, à travers une prose dont la force de représentation, capable de susciter le sentiment du réel, tient à la musicalité.

29. Ibid., p. 98-99.

30. Ibid., p. I00, p. I08-109.

3I. Ibid., p. 88. Le propos concernant l'ascendance "d'oïl» des nouvelles serait évidemment à nuancer, notamment du côté de leur matière : à côté par exemple des fabliaux-source de plusieurs récits, ou de la matrice tristanienne qui se surajoute à ces derniers dans la nouvelle VII 7 , il convient de rappeler que l'histoire de Guiglielmo Rossiglione (IV 9) est par exemple la réécriture d'une vida provençale.

32. Ibid., p. IOI.

33. Ibid., p. Iо3 (ce qui est souligné l'est par moi). Et un peu plus loin sur la même page : « [...] la novella non ha eroe; i suoi personaggi sono importanti solo nella misura in cui provocano degli avvenimenti, sono descritti in maniera dettagliata solo in quanto attraverso di loro la storia ci dà l'impressione di essere realmente accaduta. Lo stesso vale per la pittura degli ambienti e delle situazioni, messi in rilievo solo per il fatto di rendere gli avvenimenti più comprensibili o di contribuire a renderli verosimili» (de nouveau souligné par moi). 
Cette définition sera reprise en des termes très semblables dans maint autre texte de Jolles ${ }^{34}$. Même si donc le Boccace des nouvelles, nous est-il rappelé, réélabore une matière préexistante, tout son art va consister à faire comme si les événements rapportés ne s'étaient produits qu'une fois, cette fois-là, dans ces lieux et avec ces personnages désignés par leurs noms propres $^{35}$. Événements au sens premier - ce qui surgit à l'improviste et une fois seulement. Avec pour ambition de faire revivre tout entière sous nos yeux la société du Trecento — un seul monde, quoique infiniment divers, qui serait le principe de la cohésion de l'ensemble ${ }^{36}$.

On peut alors trouver réductrice la définition de Jolles, et pas uniquement parce que le chronotope des nouvelles du Décaméron ne se limite pas au XIV ${ }^{\mathrm{e}}$ siècle (il s'en faut de beaucoup), mais encore parce qu' elle l'est aussi en ce qui concerne les personnages et leurs caractères, à commencer par les deux qui encadrent le recueil ${ }^{37}$, à savoir Ciappelletto, d'un côté, et Griselda de l'autre : le premier se distinguant par ce qu'il dit et ce que cela laisse percer de son extraordinaire personnalité, la seconde comme son image inversée, symétrique, y compris dans son rapport à la parole, à l'autre extrémité du parcours, douée de la force d'âme sans laquelle la suite des événements l'affectant n'aurait guère de sens. Et si raison, sagesse et "industria» sont ensemble, avec Fortune et Amour, l'autre pointe du triangle du «jeu» décaméronien, essentiellement tripolaire, on en déduit que nombre de personnages se distingueront précisément sur ce plan-là, l'événement n'étant que prétexte à la mise en valeur de l'intelligence (pratique) des individus aux prises avec les deux forces en question. Cependant, que vise d'abord la série des passages initiaux successifs, constitution de la «brigata», éloignement de la "città tribolata», puis les deux nouvelles

34. «[...] la novella riporta un fatto o un evento in modo tale che esso generi nel lettore l'impressione di essere realmente avvenuto, e in una forma che attribuisce più importanza all'avvenimento in sé che al carattere o alla psicologia dei personaggi» (A. Jolles, La fiaba nella letteratura occidentale moderna [texte datant de 1922-1923], dans ibid., p. II7-2I9, ici p. II8-II9; souligné par moi). Ou encore : "Je dirai de manière générale, et sans entrer dans les détails, que la nouvelle toscane cherche à raconter un fait ou un incident frappant d'une manière telle qu'on ait l'impression d'un événement effectif et, plus précisément, en donnant l'impression que cet incident est plus important que les personnages qui le vivent" (Le Conte, dans A. Jolles, Formes simples, ouvr. cité, p. I73-195, ici p. I80).

35. A. Jolles, I travestimenti della letteratura, ouvr. cité, p. I04-Io8.

36. Ici aussi, le propos paraît toutefois un peu "court" : que faire en effet, pour ne citer que les cas les plus flagrants, de Cimone (V I), Natan et Mitridanes (X 3), Tito et Gisippo (X 8) ?

37. Pour un auteur qui médite une construction-livre tout à fait inédite, ces deux nouvelles sont évidemment capitales en ce qu'elles constituent un deuxième niveau interne de délimitation — au sens lotmanien — du texte considéré dans sa globalité (après l'Introduction à la première journée qui sert en fait de prologue général à l'œuvre tout entière, et la Conclusion de l'auteur) : voir I. Lotman, La structure du texte artistique [Struktura khudožestvenogo teksta, Moscou, Iskusstvo, 1970], traduit du russe par A. Fournier, B. Kresise, È. Melleret, J. Yong, sous la dir. d'H. Meschonnic, préface d'H. Meschonnic, Paris, Gallimard, 1973, p. 92-93, 303 et suiv. 
d'ouverture, qui ont pour fonction de rejeter hors champ toute transcendance susceptible de fournir aux épisodes rapportés une valeur soit mystique, soit exemplaire dans une perspective morale stricto sensu, théologiquement fondée? Écoutons Filomena, narratrice et donc personnage du récit porteur : après la première nouvelle du recueil, qui serait complètement déconcertante si elle n'était encadrée, dans des proportions qui ne se répéteront plus, de considérations sur les desseins impénétrables de Dieu, de son jugement, et à la suite immédiate de son prolongement dans la paradoxale nouvelle 2 destinée à établir la supériorité de la foi chrétienne, celle qui s'apprête à raconter à son tour s'exprime ainsi :

Per ciò che già e di Dio e della verità della nostra fede è assai bene stato detto, il discendere oggimai agli avvenimenti e agli atti degli uomini non si dovrà disdire $[\ldots]^{38}$.

La proximité des termes clés de la définition de Jolles avec les mots utilisés par la narratrice de Boccace pour nous introduire de plain-pied dans le monde des nouvelles du Décaméron, est frappante, sinon troublante (comme si Jolles n'avait fait que les reprendre comme socle premier de sa théorie du genre-nouvelle). Mais on relèvera aussitôt que la phrase de Filomena se poursuit comme suit : "[...] a narrarvi quella verrò, la quale udita, forse più caute diverrete nelle risposte alle quistioni che fatte vi fossero". Non seulement donc la portée praxéologique, fondée sur le "senno", de ce qui va être raconté, est soulignée, mais cette matière se voit dotée de la vertu d'apporter une réponse à ce qui va apparaître comme une sorte de cas d'école — "quistione» portant le souvenir de l'exercice scolaire que le mot d'ordinaire désigne. Ou, en d'autres termes, les «casi» que nous proposent les nouvelles seront porteurs d'indications utiles pour se mouvoir au milieu des aléas de l'existence, parmi ce qu'il nous faut au cas par cas débrouiller pour mieux y "répondre».

"Casi della fortuna": c'est par ce syntagme en effet qu'est annoncé en Conclusion de la première journée, $\$$ IO, le thème commun à toutes

38. Voir Dec. I 3, 3 (les mots soulignés le sont par moi; on remarquera au passage, dans le sillage de ce que nous relevions deux notes plus haut, que la nouvelle de Melchisedec et de Saladin qu'introduit ainsi Filomena n'a rien à voir avec le Trecento). La garantie de véridicité quant à ce qui a été - et donc le fait même que cela ait été —, fondatrice du "pacte" du "novellare», est rappelé par exemple par Fiammetta au début de IX 5 : «Per la qual cosa, posto che assai volte dé fatti di Calandrino detto si sia tra noi [...], ardirò oltre alle dette dirvene una novella: la quale, se io dalla verità del fatto $\mathrm{mi}$ fossi scostare voluta o volessi, avrei ben saputo e saprei sotto altri nomi comporla e raccontarla; ma per ciò che il partirsi dalla verità delle cose state nel novellare è gran diminuire di diletto negl'intendenti, in propria forma, [...] la vi dirò.» (Je cite d'après G. Boccaccio, Decameron, introduzione, note e repertorio di Cose [e parole] del mondo di A. Quondam, Testo critico e Nota al testo di M. Fiorilla, Schede introduttive e notizia biografica di G. Alfano, Milan, Rizzoli, «BUR», 20I3; les passages soulignés le sont par moi). 
les nouvelles de la deuxième ${ }^{39}$; le roi de la quatrième journée, Filostrato, au moment d'introduire la sienne, la neuvième de la série, rappelle que ladite journée porte sur les «infortunati casi d'amore» (IV 9, 3). Mais déjà dans le Proemio I4, l'auteur annonçait que ses "nouvelles" offriraient des "piacevoli e aspri casi d'amore e altri fortunati [i.e. dus à la fortune, propice ou adverse] avvenimenti [...]»(c'est moi qui souligne). La Fortune, considérée en elle-même, tend en réalité à nous proposer des "accidenti ${ }^{40}$, dans toute leur simplicité et brutalité (le premier «accident» dans le Décaméron étant au demeurant, paradigmatiquement, la peste $\left.^{41}\right)$. Sans que l'on puisse s'appuyer de façon trop rigide sur une spécialisation méthodique de l'usage en réalité fréquemment poreux des deux termes ${ }^{42}$, le "caso» (structuré le cas échéant sur une multiplicité d' "accidenti», ou de "casi» — $c f$. notamment les nouvelles de la journée II) tend parfois à annoncer une structure plus complexe. En effet, hormis pour les occurrences où il apparaît dans la locution adverbiale "per caso" ou prépositionnelle "in caso di", il met en jeu comme par définition les agents du récit en butte à la loi de la Fortune ou à celle de l'Amour (ou des deux conjuguées), appelant leurs "réponses» et leurs réactions ${ }^{43}$. Lorsque "caso" n'a pas simplement, selon la parenté étymologique, le sens d' "occasion", ou encore de "circonstance» — on remarquera cependant que le sens même d'occasion ou de circonstance implique en creux, et toutefois nécessairement, l'existence de qui est appelé à la saisir, à s'y conformer ou s'y opposer ${ }^{44}$ —, la logique du "caso" peut être celle d'un "complexe» intrinsèquement multidimensionnel, éventuellement signifié par l'emploi du mot au pluriel ${ }^{45}$.

39. Voici le thème de la journée : «incomincia la Seconda [giornata], nella quale, [...] si ragiona di chi, da diverse cose infestato, sia oltre alla sua speranza riuscito a lieto fine. " L'on relèvera le nouvel usage de "cose».

40. Le dépouillement lexical systématique d' "accidente" donne une cinquantaine d'occurrences entre singulier et pluriel dans tout le Décaméron. Rappelons que «caso " et "accidente» ont pour racine commune l'idée de la chute, de ce qui tombe.

4I. "accidente mortifero» : ainsi la peste est-elle définie en I Intro, 47.

42. Voir par exemple Andreuccio da Perugia, II 5, 40, dont le "caso " est alors plutôt un véritable «accident». Intéressants aussi les emplois contigus suivants (IV 6,3I) : «E cosí andando, per caso avvenne che dalla famiglia del podestà, che per caso andava a quella ora per alcuno accidente, furono [Andreuola et sa "fante»] trovate e prese col morto corpo."

43. Dioneo dans la Conclusion de la journée VI, $\$ 4$, rapproche «casi» et «industria» ( Valorose donne, in diverse maniere ci s'è della umana industria e de' casi varii ragionato tanto [...]»).

44. I 4, I6; III 7, 96; VIII 7, 46.

45. Nous recensons quarante-six occurrences entre singulier et pluriel. Pour ce qui nous intéresse ici, dès I 3,3 , le «dubbioso caso già avvenuto a un iudeo ", autrement dit, la conjoncture incertaine, embarrassante, ou encore "la situation hasardeuse», selon la dernière traduction en français (Boccace, Le Décaméron, traduction nouvelle et postface de G. Clerico, préface de P. Laurens, dossier de C. et P. Laurens, Paris, Gallimard, «Folio classique», 2006, p. 80). 
Mais alors, une question surgit comme malgré elle : quel rapport ces «casi», autrement dit le "cas» au sens où Boccace l'emploie la plupart du temps, pour désigner les «accidents» de l'existence, les péripéties auxquelles sont sujettes nos vies, mais aussi, plus rarement, pour caractériser en un sens plus ample les situations concrètes, toujours singulières, plus ou moins complexes que nous avons à affronter comme problèmes à résoudre, et qui sont l'objet de la mise en scène des récits du Décaméron, quel rapport, donc, cet usage du mot entretient-il avec ce que Jolles appelle dans les Formes simples le Cas ( Kasus») ${ }^{46}$ ? La question paraît encore plus fondée si l'on se souvient que ce Cas jollesien, dès lors qu'il est "actualisé», confine très vite à une espèce bien définie de «forme savante»: "cette forme, qui montre l'événement frappant dans son unicité mais pour lui-même et sans vouloir en faire un Cas [avec la majuscule de la "forme simple»], parce que précisément elle est Forme savante, nous l'appelons Nouvelle ${ }^{47}$ ».

Revenons un instant à la fin de l'Introduction de l'ouvrage en question, où se définit l'anthropologie jollesienne comme modalité de la présence au monde :

L'homme intervient dans la confusion de l'univers; il approfondit, il réduit, il réunit; il rassemble les éléments connexes, il sépare, il divise, il décompose et rassemble l'essentiel sur ses petits tas. [...] Déploiement de l'explication et repliement du classement — l'homme parvient ainsi jusqu'aux Formes fondamentales ${ }^{48}$.

Chacune de ces formes donne lieu à des « agrégations» dérivées, « formes actualisées» qui préludent elles-mêmes à des «formes savantes». Une telle activité est dès le départ construction, production langagière — à partir du langage comme travail consistant à nommer tout ce qui, à partir de l'ordre des choses tel qu'il se donne dans la nature, a été cultivé, fabriqué, interprété (selon la traduction jollesienne de la tripartition des fonctions), pour ensuite transformer et renouveler (interpréter) le fruit de ces activités : il fait ainsi acte poétique (au sens propre), conduisant ipso facto à la littérature $^{49}$. On va ainsi du Langage à la Poésie, de la «forme simple» à la «forme savante " ${ }^{5}$. Pour ce qui est de la nouvelle, ce qui l'oppose non plus au Cas,

46. L’une des neuf "formes simples" (la sixième, après la Légende, la Geste, le Mythe, la Devinette, la Locution, avant les Mémorables, le Conte, le Trait d'esprit, les Perspectives); voir A. Jolles, Formes simples, ouvr. cité, p. I37-I57.

47. Ibid., p. I45 (souligné par moi). Du reste, «le geste verbal [dans lequel se réalise le Cas] n’est pas si contraignant, il ne s'empare pas des choses avec la même sûreté absolue que dans les autres formes simples» (ibid.).

48. Ibid., p. 26. Sur la conception du langage comme travail (et l'anthropologie jollesienne qui en est le socle), voir ibid., p. I8-25.

49. Voir en particulier ibid., p. 23.

50. Ibid., p. I80. 
mais à l'autre «forme simple» qu'est le Conte, c'est que sa tâche consiste à appliquer sa norme à une diversité infinie de faits librement choisis, en les représentant sous la forme définitive et absolue que leur a donnée l'artisteécrivain. Car «nous pouvons par les forces de notre imagination fabriquer une construction littéraire qui incarne sous une telle forme cette portion d'univers de manière autonome et hors de tout cadre ${ }^{5 \mathrm{I}}$.

Pour en revenir alors à la question du rapport de la nouvelle avec le Cas, en quoi se distingue-t-elle de celui-ci? Sur la trame d'une sorte de squelette, elle s'alimente de l'ensemble des additions inessentielles (interchangeables), de tout ce qui ne résulte pas directement de la «forme simple» du Cas et qui lui confère son unicité et sa force d'impact. Mais cela, le Cas, à la différence des autres «formes simples», le prédispose, paraît l'appeler $a b$ initio. Non seulement le Cas contient en germe ses développements à venir, mais ceux-ci sont en quelque sorte inéluctables : avec pour point de fuite la forme aboutie de la nouvelle ${ }^{52}$.

Il faut en même temps toujours avoir bien présent à l'esprit que le Cas comme "forme simple», proposant une situation théorique se configurant comme "devoir de décision", est en tant que tel anéanti par le "cas" boccacien qui, lui, est tout entier tendu vers ce qui constitue précisément sa cause finale, à savoir le moment de la décision, la décision elle-même. Toujours différent, irréductible, c'est le moment de l'actualisation dans ce qu'il a d'absolu qui constitue le geste originel : la nouvelle détruit alors le Cas dont elle est l'amplification ${ }^{53}$. Et ce, même si quelque chose comme une parenté originelle subsiste, en ce sens que le "cas» décaméronien entretient dans nombre de nouvelles, explicitement ou implicitement, un lien avec le judiciaire, et parfois de manière étroite avec la norme de la loi, la faute et la punition ${ }^{54}$.

Mais, par-delà les incarnations du jugement social internes aux récits, souvent collectives ss, le lieu par excellence de l'instance jugeante, à la fois au sens esthétique et au sens éthique (les nouvelles comme échantillons de l'infinie diversité des comportements humains) est la "cornice»; instance

5I. Ibid., p. I84; quelques lignes plus loin p. I84-I85: «les lois de "formation" de la nouvelle sont telles qu' elle peut donner un visage cohérent à tout incident, rapporté, réel ou inventé, pourvu qu'il ait pour caractéristique spécifique d'être frappant».

52. Ibid., p. I43-I44.

53. Ibid., p. I5I, I54. "Amplification" n'est pas à entendre ici en un sens seulement quantitatif, mais comme déploiement de ses potentialités.

54. Ibid., p. I39-I40. Paradigmatique à cet égard apparaît la célèbre nouvelle IV 7 (Simona et Pasquino), dont la leçon principale, sur ce plan-là, est que les situations rencontrées par un juge sont étonnamment diverses, irréductibles les unes aux autres (mais voir aussi IV 6, etc.).

55. Exemplaire de ce point de vue nous paraît l'analyse d'E. Auerbach, Mimésis, ouvr. cité, p. 230. 
qui, en réalité, dès lors que la décision est prise de faire livre, précède dans l'ordre non seulement narratologique, mais simplement logique : concrètement, une telle instance est représentée par les membres de la "brigata», figure plurielle du récepteur (auditeur, puis lecteur), mais d'un lecteur qui, s'il cherche à conjuguer pour son plus grand profit - double profit plaisir et leçons de vie, ne cherche pas l'édification proprement dite. Ce n'est donc pas le «cas" d'une casuistique juridique, déterminant a priori jugement (de la faute) et sanction. Tel était aussi déjà, sous l'angle d'une certaine analogie, selon le Jolles de La fiaba nella letteratura occidentale moderna, le sens du passage des "cas» constitués par les questions d'amour du livre IV du Filocolo aux nouvelles du Décaméron, en particulier celles qui en dérivent directement ${ }^{56}$. Du problème théorique au fait concret, à l'événement se présentant comme vrai, unique et autosuffisant : il n'y est plus guère question de norme et de mètre, et si l'on évalue et tranche, on ne juge à proprement parler ni ne punit ${ }^{57}$. L'évaluation des situations concrètes, comme les solutions, sans cesse renouvelées au gré de l'inventivité de ceux qui mobilisent en fonction des occasions et circonstances (des "casi» qui leur échoient) les ressources de leur "ingegno", de leur "senno", de leur «intelletto", de leur «saviezza» ou "discrezione», de leur «industria» ou de leur promptitude d'esprit, enfin, sont fonction d'une morale toujours provisoire qui se forge au fur et à mesure des besoins qui, même si c'est une morale problématisée, immanente, non «moralisatrice», n'en est pas moins une morale. Et qui, dans les nouvelles, concerne au premier chef les individus que nous sommes, nous offrant à l'occasion "une vérité nécessaire, dont le contenu tragique ou comique manque à notre vie». Ou, plus précisément :

Quando la verità ci offre un'immagine riflessa di ciò che noi stessi stiamo vivendo, passiamo oltre con indifferenza, mentre se ci consente di colmare una lacuna ci fermiamo su di essa. Così nella novella erotica l'amore non è quello che possediamo, ma quello che ci manca. Amore non è l'immagine divina di ciò che ci appartiene, ma ciò che desideriamo avere.

56. Il s'agit de Dec. X 4 et 5 et Filocolo IV 3 I et 67; voir aussi A. Jolles, I travestimenti della letteratura, ouvr. cité, p. II9-I2I. Paolo Cherchi, par un autre biais, souligne le rapport qu'entretient le genre avec les formes traditionnelles du judiciaire, en s'attachant au lien natif de la nouvelle avec la «question" qui est à l'origine de l'acte de juger dans la controverse : voir From Controversia to Novella, dans La Nouvelle, ouvr. cité, p. 89-99.

57. Voir A. Jolles, Formes simples, ouvr. cité, p. I54. Voir aussi, par exemple, dans le cadre d'une mise en perspective liminaire sur la question de la nouvelle occidentale en général, les observations sur le Décaméron d'Enrico Malato, La nascita della novella italiana: un'alternativa letteraria borghese alla tradizione cortese, dans La novella italiana, ouvr. cité, p. 3-45, pour ce qui nous intéresse ici, p. 24-27. 
C'est la raison pour laquelle ces histoires apparaissent comme vraies non parce qu'elles sont telles, mais parce que nous - chacun d'entre nous - les écoutons volontiers ${ }^{58}$. Tout en nous faisant voir, effet paradoxal, derrière la différenciation, l'instable et le mouvant, ce qui reste immuable — inchangé comme le baiser d'Alatiel ${ }^{59}$ —, à savoir la libido narrandi et ce qui la motive - cette pulsion à la mise en récit comme corollaire de l'appétit sous toutes ses formes (à commencer par l' "appetito concupiscibile»).

Boccace réussit donc ce faisant à réunir dans une unique symphonie, produite par une subjectivité plurielle mais concordante, les différentes tonalités, à concilier dans un ensemble harmonieux les contradictions ${ }^{60}$. Bref, si l'on en revient au niveau auctorial, il parvient par le biais de la délégation ${ }^{6}$, à tenir sous l'égide de sa personnalité propre d'écrivain ses nouvelles, traversées qu'elles sont par les deux grandes forces (les «idoles») que sont Fortune et Amour ${ }^{62}$. L'on remarquera ici en passant, que c'est sous ce double sceau (appelant la réponse «industrieuse» de la raison) que s'inscrit déjà l'histoire de la "brigata»: la Fortune, ayant les traits de la peste, dont il est difficile d'imaginer manifestation plus puissante; les jeunes gens en proie, quoique sur un mode éminemment urbain (Dioneo compris, qui est là pour indiquer la frontière), aux affects dérivant de la passion amoureuse - objet au demeurant des ballades injustement négligées jusqu'à une période récente ${ }^{63}$. Nous avons donc affaire à $u n$ monde de l'œuvre, comprenant le "roman de la brigata». C'est à l'intérieur de celui-ci, ouverture d'espace et fond plutôt que cadre, que se constituent les strates et les passages, les seuils qui relient ces différents niveaux entre eux et permettent d'arriver en toute légitimité et liberté (contrôlée) aux atti e avvenimenti que l'on veut pouvoir rapporter sans frein aucun. Car nous rappelions plus haut que la «cornice» est le lieu de l'ordre (de la

58. Pour tout ce passage, citation comprise, voir A. Jolles, I travestimenti della letteratura, ouvr. cité, p. II2.

59. Ibid., p. Io8.

6o. Ibid., p. I09-IIo.

6I. La formulation de saveur juridique a pour fonction d'indiquer que si les énonciateurs sont les différents narrateurs et engagent en tant que tels leur responsabilité, la responsabilité ultime repose sur l'énonciateur premier, dans une relation refondée avec ses récepteurs (voir la justement célèbre Conclusion de l'auteur).

62. Voir A. Jolles, I travestimenti della letteratura, ouvr. cité, p. IIO-II2.

63. Voir R. Zanni, «La "poesia” del Decameron: le ballate e l'intertesto lirico ", Linguistica e letteratura, XXX I-2, 2005, p. 59-I42; Ph. Guérin, Le Décaméron, un prosimètre sui generis?, dans C. Croizy-Naquet, M. Szkilnik (éd.), Rencontres du vers et de la prose. Conscience théorique et mise en page, Turnhout, Brepols, "Texte, Codex \& Contexte», 20I5, p. 9I-IO4. Mais nous rencontrons à ce sujet une autre intuition étonnante de Jolles : c'est là, avec les ballades du Décaméron, que selon lui Boccace se montre véritablement poète (I travestimenti della letteratura, ouvr. cité, p. 68). 
mesure, de la ratio) qui s'oppose au désordre du monde ${ }^{64}$. Mais c'est qu'il s'agit in ultimo de conjuguer, pour une opposition efficace, "ordre et plaisir» (I Intro, 98), "ordre et liberté» : cadre qui va se décliner dans l'infinie variété du "novellare», des situations concrètes qu'il décrit, visant le retour à l'équilibre (sur le plan narratologique), qui est généralement restauration de l'ordre perturbé par une prise de liberté mal comprise ou une libido effrénée ${ }^{65}$. C'est aussi le sens métapoétique de la conclusion de la huitième journée, en ses paragraphes centraux : liberté, mesurée dans le temps et dans l'espace (et, par métaphore, dans l'espace textuel, bien entendu), et variété (avec le corollaire d'une telle diversité des choses et de leurs qualités, l'indispensable pulsation de la variation) sont les clefs de la vie heureuse selon le Boccace du Décaméron ${ }^{66}$. De tout cela, le récit-cadre constitue la condition de possibilité, telle que l'incarne «à la limite» le personnage de Dioneo ${ }^{67}$. Tel est le grand facteur de cohésion sur le plan esthétique - toujours au premier plan dans le Décaméron, tout en restant indissociable du plan éthique. Mais ce droit à la liberté ne se donne que

64. Rappelons l'appel lancé par la sage Pampinea, au moment de constituer la «brigata», à "onestamente usa[re] la sua ragione», à respecter la "natural ragione» (I Intro, 53), à ne pas outrepasser le "segno della ragione» lorsque l'on prend du plaisir (I Intro, 65); la satisfaction d'avoir sans faillir observé l'«honnêteté»" requise par les circonstances (X Concl., 4-5), véritable leitmotiv au demeurant du métadiscours du récit porteur.

65. Mais que liberté (éventuellement sexuelle) et plaisir ne soient pas inconciliables avec la mesure, c'est ce que nous rappelle constamment le commentaire des narrateurs : voir les conclusions de I 4 (la première nouvelle du licencieux Dioneo, et l'usage final paradoxal de l'adverbe «onestamente»), III 3, 6, 7, 8 (où l'on rencontre de nouveau «onestamente»), Io, VII 4, etc.; ou l'introduction de II 3, VI 8, IX 7 et passim.

66. Voir VIII Concl., 3-5: «Dilettose donne, assai manifestamente veggiamo che, poi che i buoi alcuna parte del giorno hanno faticato sotto il giogo ristretti, quegli esser dal giogo alleviati e disciolti, e liberamente dove lor piú piace, per li boschi lasciati sono andare alla pastura: e veggiamo ancora non esser men belli ma molto piú i giardini di varie piante fronzuti che i boschi ne' quali solamente querce veggiamo; per le quali cose io estimo, avendo riguardo quanti giorni sotto certa legge ristretti ragionato abbiamo, che, sí come a bisognosi, di vagare alquanto e vagando riprender forze a rientrar sotto il giogo non solamente sia utile, ma oportuno. E per ciò quello che domane, seguendo il vostro dilettevole ragionar, sia da dire non intendo di ristrignervi sotto alcuna spezialità $[\ldots]$ ». Pour ce qui est du motif de la variété, et de celui corollaire (ici bien entendu "déthéologisé») de la nécessaire imperfection, mais complémentarité des choses de ce monde, on peut rapprocher ce passage de celui qui dans la Conclusion de l'auteur, I7-I8, concerne de façon plus explicitement métadiscursive l'«oste» de Charlemagne qui ne compte pas que des paladins.

67. Le rôle de Dioneo est depuis longtemps au centre de l'attention. Citons par exemple, parce que le propos coïncide avec la visée poursuivie ici, F. Bruni, Boccaccio. L'invenzione della letteratura mezzana, Bologne, Il Mulino, 1990, p. 237; ou L. Surdich, Boccaccio, Rome-Bari, Laterza, "Scrittori italiani», 200I, p. IO6 : «A Dioneo [...] viene riconosciuta una facoltà di trasgressione che non è però rinnegamento od opposizione al gruppo: è trasgressione concessa dal gruppo e dal gruppo stesso controllata. Lungi dallo smentire il sistema, Dioneo lo conferma [...]. Dioneo è l'eccezione consentita che rafforza l'unità della brigata, proprio perché la sua libertà non è irruzione di un elemento arbitrario e incontrollabile, ma è una libertà concessa, contemplata dalle regole istituzionali su cui il gruppo dei dieci giovani fonda la propria esistenza.» Dans une perspective auerbachienne, on remarquera que Dioneo se fait aussi vecteur de la non-séparation des styles, de leur contamination, ou en tout cas, de leur perméabilité (relative, non absolue). Voir encore sur ce point, bien évidemment, la Conclusion de l'auteur. 
dans l'équilibre dialectique entre les deux termes du binôme, équilibre subtil et fragile, toujours en tout cas à refonder.

Si la nouvelle est l'acte par excellence du refus de l'allégorie, en ce qu'elle met en scène un événement se présentant comme vrai, unique et autosuffisant, elle n'est pour autant pas dépourvue de signification. Il y a une pragmatique propre de la nouvelle et elle peut bien s'opposer en première analyse à celle de la "cornice», celle-ci ornementale, celle-là purement «réaliste», à l'honnêteté d'une «lieta brigata» aristocratiquement protégée par son entre-soi contre l'entropie du monde réel. Mais en vérité, c'est le cadre «décoratif» qui finit par correspondre à la réalité, tandis que les nouvelles, d'abord perçues comme réelles, sont un révélateur d'idéalités $^{68}$. Jolles pousse ici son raisonnement jusqu'au bout et anticipe la thèse fameuse de Branca (quoiqu'à nos yeux largement contestable) d'un parcours rigoureusement ascensionnel, d'un «triomphe» défini comme menant "de la Fortune à l'Amour, de l'Amour à la Magnanimité ${ }^{69}$ ». Mais c'est pour y voir aussi le principe d'unité suivant, constituant la fin visée par sa démonstration, annoncée d'emblée ${ }^{70}$ :

[...] in questo modo nel testo di Boccaccio due antiche forme della poesia, la cornice e il racconto a catena confluiscono in una sola, che rivela le sue profonde radici nella cultura popolare, e io non sono venuto meno alla promessa iniziale di dimostrare che anche il Decameron è un libro morale ${ }^{71}$.

Nous réaffirmons toutefois notre scepticisme sur le «motif de la progression", et du "dépassement» [overwinnen en néerlandais] dans la dernière journée, de tout ce qui précède sans pour autant l'effacer, l'annuler. Socle tout autant hégélien que "populaire», sans doute... Car Jolles oublie la réplique de Dioneo-narrateur (et figure de l'auteur, nous disait-il luimême plus haut), par quoi il conclut son récit édifiant, son prétendu portrait d'idéal féminin, opposé à des degrés divers à toutes les autres femmes - la Griselda de la toute dernière nouvelle ${ }^{72}$. Comme si Ghismonda, Filippa et quelques autres n'étaient pas elles aussi des types "idéaux", susceptibles du reste d'illustrer la thèse tout juste évoquée de la nouvelle comme révélateur d'idéalité. Il ne faut alors pas se méprendre sur le sens de «morale». Même si Jolles ne le formalise pas ainsi, c'est lui encore qui nous le suggère : à leur façon également, les nouvelles ont pour fonction

68. Selon A. Jolles, I travestimenti della letteratura, ouvr. cité, p. II3.

69. Ibid., p. II4. Et pour Branca, voir son Boccaccio medievale, ouvr. cité.

70. Voir plus haut la n. 22 (A. Jolles, I travestimenti della letteratura, ouvr. cité, p. 65).

7I. Ibid., p. II4.

72. Ibid., p. II5. 
de contrer le chaos du monde. Relevons qu'il y a là, nous semble-t-il, dans le traitement du "caso» comme réponse, un autre point de jonction avec la problématique jollesienne du Cas en son essence même, et qui ferait de la nouvelle une structure traversée ab initio par la dialogicité du rapport de l'homme au monde et à ses semblables ${ }^{73}$. La résolution du "cas", et donc sa disparition en tant que tel, dans l'acte final de la nouvelle, ne doit pas nous faire oublier que c'est dans la tension extrême d'une sorte de dialogue que se prépare, voire se rend nécessaire, l'abolition de l'ouverture initiale. Le récit porteur en son fondement même peut à cet égard servir de paradigme : il est loisible en effet d'analyser la grandiose introduction à la première journée comme réponse à un Cas, au sens de Jolles, aux questions auxquelles il presse instamment ceux qui y sont confrontés de répondre : pendant la peste, est-il ou non licite d'abandonner sa ville en proie au deuil et à la dissolution des mœurs pour aller passer du bon temps à la campagne (et peut-être sauver sa vie, mais sans que cela soit certain)? Avec redoublement, mise en abyme de l'actualisation dans et par le récit: car la réponse coïncide avec la possibilité qui s'y donne d'y mettre en récit le monde des hommes, leur «comédie».

Un dernier point, enfin, concernant la problématique de l'actualisation. Un très beau passage de l'essai sur le Décaméron nous paraît annoncer les interrogations contemporaines sur les valeurs (valences) du transcodage et de la transtextualité, lorsque le savant hollandais s'interroge sur les sources de Boccace auteur de nouvelles et part de l'hypothèse d'une origine des récits lui échappant certainement (Jolles croit qu'il s'agit d'une très large majorité des $\mathrm{cas}^{74}$ ). À l'instar de ce qui arrive au commis-voyageur, au colonel à la retraite ou à la vieille tante pour ce qui est de leurs propres

73. "le Cas est, lui aussi, lié à la question», voir A. Jolles, Formes simples, ouvr. cité, p. I5o. N'oublions pas, Dec. I 3, 3, qu'il s'agit pour les jeunes femmes de la «brigata» de devenir plus prudentes et avisées ("caute») dans leurs "risposte alle quistioni che fatte [loro] fossero", en prenant connaissance des "fatti e [...] avvenimenti degli uomini». Ce qui apparenterait, en termes jollesiens, la nouvelle du Mythe et de l'Énigme (voire de la "Locution» et des "Mémorables»), en nous faisant approcher une composante essentielle de la présence de l'homme au monde (quitte donc à devoir réduire sans doute le nombre des "formes simples») : voir F. Fonio, «Dell'applicabilità del sistema delle Einfache Formen di André Jolles. Una tappa della tematologia nella prima metà del Novecento", Franco-Italica, 29-30, 2006, p. 199-227, ici plus particulièrement p. 202-203, 205, 219220. La structure dialogique de la nouvelle pourrait-elle alors être aussi appréhendée comme une sorte de préfiguration de ce qui constitue l'essence du roman, vue au prisme de la théorie romantique (sinon aussi bakhtinienne) de celui-ci? Nous pensons ici au Schlegel des Fragments (que l'on peut lire en français dans Fr. Schlegel, Fragments, traduit et présenté par Ch. Le Blanc, Paris, José Corti, 1996), qui considère que «les romans sont les dialogues socratiques de [son] époque", fr. 26 (Jolles cite dans La fiaba nella letteratura occidentale moderna, dans Id., I travestimenti della letteratura, ouvr. cité, l'oncle Johann Elias et le frère August Wilhelm, mais pas Karl Wilhelm Friedrich). Et en tout cas la nouvelle, Kunstform première dans les grands projets théoriques initiaux jollesiens, comme nous le rappelle opportunément S. Contarini dans " $F o r m e$ artistiche": Jolles e la teoria della novella», art. cité, p. Iо9.

74. A. Jolles, I travestimenti della letteratura, ouvr. cité, p. 78. 
sources (le concept même de source du coup, qui en vient à se diluer, est mis en question $\left.{ }^{75}\right)$. Ces récits premiers sont parvenus, nous dit-il, là où on les trouve sans bien savoir comment et, au départ, ils apparaissent comme "sauvages ${ }^{76}$. Les belles lignes qui suivent, très datées ${ }^{77}$, mais révélatrices à la fois de l'ambition et de la méthode de l'auteur, méritent, croyons-nous, d'être citées in extenso :

Dipendenti dalle singole condizioni di vita, si sviluppano in generale senza regola, ma alcuni vengono coltivati, educati o plasmati dal mezzo artistico. Di qui nasce la fermentazione vera e propria del racconto, quando le storie vengono dotate di una struttura e di altri elementi formali come il contrappunto, il ritardo, la tensione, la sorpresa; infine, stile e arte poetica conferiscono loro forma e tono, e in questo modo si inseriscono ufficialmente vicino al canto e alla danza, oppure al dramma, al romanzo o all'epos. Se trascurati, ritornano allo stato selvaggio: si affrancano dalle forme artistiche, si nascondono in un angolo buio che non ha nulla a che fare con la letteratura, al punto che noi stessi, anche quando li abbiamo incontrati come la brillante prestazione di un maestro dell'arte del racconto, o l'opera di un poeta di genio, stentiamo a riconoscerli se li ritroviamo sotto forma di narrazione grezza nella camera di un bambino o mascherati da barzelletta licenziosa al bancone di osteria di uno sperduto villaggio di campagna. Tuttavia né lo sviluppo artistico né il loro ritorno allo stato selvaggio possono trasformarne la sostanza al punto da renderli irriconoscibili. Per quanto appaiano sovraccarichi di ornamenti e di fronzoli, oppure, al contrario, contratti, disgregati o consunti, il nucleo originario che rivela la loro tipologia rimane identico. E, cosa ancora più stupefacente, né nella buona sorte né nella cattiva le tipologie si moltiplicano o si riducono: pure qui infatti regna sovrana la legge delle possibilità limitate ancora troppo poco studiata e definita nella storia dell'arte.

In un angolo recondito della mente dell'uomo comune si trova celato un giardino, dove insieme a proverbi, detti, favole e simili fiorisce una flora più o meno abbondante di racconti. A un primo sguardo sembrano molto diversi tra loro, una profusione di colori: una parte non appartiene alla tradizione scritta, alcuni ci sono stati letti da bambini, altri li abbiamo imparati in qualche forma a scuola, tramite l'insegnamento delle lingue antiche o moderne, altri ancora li abbiamo scoperti più tardi nella letteratura o nel teatro. Ma nel tempo si sono affrancati del tutto o in parte dalle forme nelle quali li abbiamo appresi, e si conservano dentro di noi come storie e nulla di più ${ }^{78}$.

La citation n'a pas tant pour visée de montrer Jolles en quête de la "forme simple», que d'une sorte d'archétype de "forme actualisée», en

75. Ibid., p. 78 .

76. Ibid., p. 76-77.

77. «L'impianto di Jolles congiunge un'ispirazione ancora romantica (ricerca delle radici, concetto di poesia popolare e poesia d'arte) con intuizioni formalistiche sorprendenti anche se non maturate» (C. Segre, Avviamento all'analisi del testo letterario, Turin, Einaudi, [1985] 1999, p. 252).

78. A. Jolles, I travestimenti della letteratura, ouvr. cité, p. 77. 
d'autres termes, de quelque chose de très proche sans doute de ce que le Lotman théoricien du «transcodage pragmatique» désigne comme «système de modélisation primaire" préludant au "texte artistique», "système de modélisation secondaire " 79 . Or, l'actualisation est d'abord dans le Décaméron celle de l'énonciation par le biais du récit porteur. Ses histoires, les nouvelles qu'il contient, nous sont contemporaines d'abord parce que se les approprient les jeunes gens de la "brigata», "coetanei», ou peu s'en faut, de celui qui les transcrit, et qui se les racontent pour que, génération après génération, nous les entendions comme eux les ont entendues. Comme eux : évidemment pas avec les mêmes grilles de lecture, le même horizon de réception, mais, comme dignes héritiers de ceux qui sont notre figure archétypique, dans le procès d'actualisation sans cesse renouvelé en quoi consistent nos propres lectures, nos appropriations successives, à la fois plaisantes et utiles.

Nous nous acheminons alors vers notre conclusion.

On souligne désormais volontiers, voire assez systématiquement le rôle du récit porteur par rapport au macrotexte, pour lui assigner une grande fonction métatextuelle, en particulier celle de «filtre», qui va bien audelà de la fonction ornementale déjà évoquée au début de ces pages, et consistant à mieux faire ressortir les nouvelles, à les mettre en lumière ${ }^{80}$. Francesco Bruni, par exemple, y consacre quelques remarques heureuses ${ }^{81}$. Revendiquant (selon l'interprétation du Proemio, I3) le caractère composite de ses nouvelles, Boccace, qui construit un livre, recherche le principe centripète susceptible de faire tenir solidement l'édifice. Et pas seulement, donc, comme ossature, mais comme "filtre», précisément, entre l'écrivain (ou plutôt le narrateur principal, métadiégétique) ${ }^{82}$ et la matière

79. Voir I. Lotman, La structure du texte artistique, ouvr. cité, en particulier p. 36-37.

8o. Voir G. Getto, Vita di forme e forme di vita nel Decameron, ouvr. cité, p. 33 : "La pulsante vita germogliata dalla curiosa memoria dello scrittore viene così come filtrata dal cristallo nitido e uguale della cornice [...]». Et L. Battaglia Ricci, dans Boccaccio, ouvr. cité, p. I55 : «[...] il novellare è parte di una civile conversazione: attraverso la voce dialogica della brigata, che funge da filtro ideologico, la parola [...] diventa mezzo per una rifondazione mentale e razionale del mondo distrutto dall'evento apocalittico.» (Dans les deux citations, c'est moi qui souligne).

8I. Voir F. Bruni, Boccaccio, ouvr. cité, p. 235-240. Voir aussi pour la dialectique de l'«anarchie» et de la norme, en vue de la remise en ordre du monde (la grande "fonction" assignée à la "brigata", permettant de rendre compte des "contradictions" de la "cornice» — par exemple le refuge cherché dans le "contado", pourtant pas davantage épargné par la peste que la ville), G. Bàrberi Squarotti, Il potere della parola, ouvr. cité, et plus particulièrement, pour le rapport entre récit-cadre et nouvelles, p. 46-48 (pour le "privilège» de Dioneo) et 56 et suiv. (où l'auteur envisage à sa manière la question du "Cas").

82. Et en réalité, il y a deux régimes distincts de cette diégèse englobante, puisque le narrateur principal, spectateur direct de la peste, se dédouble à partir du $\$ 49$ de l'Introduction à la journée I, pour rapporter ce qu’il a entendu d'une "persona degna di fede" : autre passage, autre effet de seuil, qui renforce la fonction "filtrante" assignée au récit-cadre. 
hétérogène de ses nouvelles, tenues à la juste distance. Filtre constitué de frontières textuelles, dont on rappellera que le concept prévoit à la fois la séparation et la jonction, la possibilité même du passage.

Mais on a envie d'aller plus loin encore. Jolles, malgré ses approximations $^{83}$, nous indique la voie pour reprendre et approfondir la question en échafaudant le cadre général à l'intérieur duquel, par exemple, des intérêts nouveaux, comme celui porté à la question des ballades qui occupent une place centrale dans les conclusions des journées et des liens qu'elles entretiennent avec la matière narrative, nouvelles comprises, trouveraient leur lieu propre en relation avec la systématique de l'ensemble ${ }^{84}$. Et ce n'est pas non plus l' "applicabilité»" ${ }^{85}$ de la sixième "forme simple» comme socle archéologique de la nouvelle et voie d'accès à la compréhension de sa genèse qui constitue notre horizon présent, mais, à l'intérieur de la "pensée-Jolles», ce qui assure la cohérence de sa lecture du chef-d'œuvre de Boccace et, par-delà, nous offre une clé pour mieux appréhender la profonde unité de la "machine-Décaméron», l'intime solidarité de ses constituants fondamentaux.

Nous ne considérons plus comme entrée pertinente la thèse de l'autobiographisme qui, partant d'une lecture du Proemio pris pour argent comptant, nous fait remonter en arrière vers l' "origine", à savoir l'amour (forcément poétique et pur) pour Fiammetta et ses traductions littéraires de jeunesse, dans le but, au terme de l'enquête, de situer Boccace à la place qui lui revient, celle du précurseur (du "style de la Renaissance»), à l'intérieur de la grande question historiographique qui le préoccupe ${ }^{86}$. Même si cet aspect de la réflexion jollesienne, qui le voit dialoguer intensément notamment avec son ami Huizinga, s'inscrit dans un chapitre passionnant de la réflexion sur Moyen Âge et Renaissance, il ne nous paraît

83. À titre d'exemple, voir A. Jolles, Il Decameron di Boccaccio, dans Id., I travestimenti della letteratura, ouvr. cité, p. 67, à propos du manque chez Boccace du "geniale istinto filologico che contraddistingue tanti umanisti della sua epoca e della successiva [c'est nous qui soulignons]" : en dehors de Pétrarque, qui peuvent bien être ces si nombreux humanistes? Ou encore La fiaba nella letteratura occidentale moderna, dans ibid., p. I2I sur le sens de I I et de son prologue («breve considerazione sulle meraviglie divine», dit Jolles, alors que c'est d'assez loin le plus important prologue à une nouvelle de tout le Décaméron, et qu'un épilogue encore plus inédit lui fait écho en fin de récit).

84. Voir les essais de R. Zanni et Ph. Guérin déjà cités.

85. Voir F. Fonio, "Dell'applicabilità del sistema delle Einfache Formen di André Jolles», art. cité. Sur cette applicabilité douteuse, voir aussi C. Segre, Avviamento all'analisi del testo letterario, ouvr. cité, p. $25 \mathrm{I}-253$.

86. On pourrait ici reprocher à Jolles de mélanger ce qu'il appelle la méthode visant au sens et à l'interprétation (le classement historique des poètes de génie, fruits du milieu et du talent) et méthode morphologique d'inspiration goethéenne, de ne pas distinguer en somme la deuxième des grandes directions des études littéraires de la troisième, celle qu'il veut fonder (A. Jolles, Formes simples, ouvr. cité, Introduction, p. I2-I5). 
pas aujourd'hui le plus fécond ${ }^{87}$. Réponse, avons-nous souligné dans le sillage de Jolles, mais pas d'abord aux aléas et aux adversités du parcours existentiel déceptif de l'auteur (Boccace). Réponse plutôt aux "cas» que nous soumet notre immersion dans le monde, aux deux niveaux rigoureusement articulés entre eux du récit porteur (du "roman", ou peut-être mieux, de la méta- et méga-nouvelle de la «brigata») et des nouvelles ellesmêmes ${ }^{88}$. C'est cette dimension fondamentale de la réflexion jollesienne qui nous permet aussi de mieux saisir l'intérêt d'un Jauss pour l'homme « interrogateur» qui se profile à l'horizon du «cas» et de sa grammaire. Un tel homme est à la fois celui qui doit directement trouver une solution pour son propre compte, mais aussi celui qui évalue la réponse, narrateurauditeur de la "brigata", c'est-à-dire en fait l'ensemble des lecteurs qui en actualisent l'interprétation ${ }^{89}$.

C'est aussi dans ce cadre qu'il est possible sans doute d'apporter une réponse au problème du genre-nouvelle et à l'obsession de la reductio ad

87. Voir A. Jolles, Il Decameron di Boccaccio, dans Id., I travestimenti della letteratura, ouvr. cité, p. 65, pour une série d'énoncés qu'il serait difficile, non pas seulement de prononcer, mais même de concevoir aujourd'hui : "Se vogliamo cogliere lo stile del Rinascimento, dobbiamo precisare anzitutto che Venere è nemica delle Muse. Chi dedica la sua vita interamente all'amore non può diventare un grande artista [...]». Et pour ce qui est du rapport à l'Antiquité (en l'occurrence l'Hellénisme), voir ibid., p. 79 et suiv. Mais on relèvera au passage que la réflexion sur les catégories historiographiques (Renaissance ou pas, et la place à assigner à Boccace) se résout en une mise au jour de l'invariant, l'Hellénisme comme «fermento dell'animo che tutto penetra, e per quanto formato da elementi molto lontani e diversi tra loro si manifesta tuttavia come uno spirito unitario" (ce qui fait du Décaméron une œuvre "contemporaine» des idylles de Théocrite et des églogues de Virgile; ibid., p. 82). Déjà auparavant (ibid., p. 8I), Jolles avait écrit que «l'universalità della forma si rivela alla fine più importante della differenza nel contenuto religioso" (entre Antiquité, Moyen Âge et Renaissance), et qu'en fait : «Nulla è qui "rinato", poiché nulla in realtà era morto, e neppure dormiva o era fiorito di nascosto, ma semplicemente si è sviluppato, e si manifesta nel luogo che con un poco di intuito storico avremmo potuto aspettarci." En d'autres termes, l'invariant ne peut réapparaître que dans un contexte historique précis - ou comment réarticuler histoire et permanence... Voir aussi ibid., p. 84 sur le toujours nouveau et le trésor des survivances.

Voir aussi l'essai sur Dec. VI 9, La facezia di Guido Cavalcanti, dans Id., I travestimenti della letteratura, ouvr. cité, p. 44-56, ainsi que l'essai de S. Contarini, Gesti verbali: Jolles lettore di Decameron, VI, 9, dans A. Ferracin, M. Venier (éd.), Giovanni Boccaccio: tradizione, interpretazione e fortuna. In ricordo di Vittore Branca, Udine, Forum, 20I4, p. 229-24I.

88. Voir A. Jolles, La fiaba nella letteratura occidentale moderna, dans Id., I travestimenti della letteratura, ouvr. cité, p. I2I : "Se ci soffermiamo sulle [...] novelle del Decameron, vedremo che alcune possono essere intese come una risposta a una certa domanda, come l'esempio o l'illustrazione di una certa tesi. »

89. Outre la place que trouve Jolles (pour la nouvelle, principalement) dans le panorama que trace Jauss dans Littérature médiévale et théorie des genres, art. cité, p. 37-76, en particulier p. 46, c'est dans le chapitre "Adam interrogateur" que le chef de file de l'herméneutique littéraire cite à plusieurs reprises le Jolles des Formes simples - la forme ici privilégiée, première, étant celle du Mythe : voir H. R. Jauss, Pour une herméneutique littéraire, traduit de l'allemand par M. Jacob, Paris, Gallimard, 1988, p. 40-42. Voir aussi ibid., p. 70-7I : «Aux questions qui se posent dans la vie de tous les jours, il y a plusieurs sortes de réponses : la positive comme dans les exempla et la fable, l'ironique dans les proverbes, la plaisante dans la farce, et celle des casuistes [souligné par moi] dans la nouvelle. Alors que les collections d'exempla tiennent prêtes et en bon nombre des réponses éprouvées pour les questions de la vie pratique sous forme de précédents historiques, le proverbe donne une leçon qui ne peut plus être utile, elle arrive toujours trop tard. [...] La casuistique est une forme plus compliquée qui ne pose la question que pour obliger le lecteur à considérer lui-même selon quelle règle il doit juger.» 
unum. Comme faux problème, vraisemblablement : car la nature même de la nouvelle pourrait bien résider dans l'infinie multiplicité des cas et leur irréductibilité à une mise en schéma uniforme. Indissociable de la multiplicité des types qu'elle englobe et transforme («fables, ou paraboles, ou histoires"), un cas risque en outre d'en cacher toujours un autre ${ }^{90}$. Le questionnement est toujours susceptible d'être rouvert. À chaque fois individualisé : ce qui en littérature correspond à la naissance de la nouvelle aurait alors en peinture un équivalent possible dans l'émergence du portrait, en tant que représentation d'un individu particulier, saisi dans sa singularité, mais au sein d'un groupe et à l'intérieur d'une architecture ${ }^{91}$.

Ce qui se dessine donc chez Jolles lecteur du Décaméron, en tout cas ce qu'il me suggère, c'est la possibilité précisément de penser l'unité du Décaméron sans avoir à le soumettre à une telle reductio ad unum. Boccace dans son ouvre maîtresse ouvre et délimite un espace légitime pour la représentation de la vie dans son absolue contingence et son irréductible diversité. Et pour les réponses à inventer de fois en fois, par le biais de l'intelligence concrète de chaque situation particulière et du langage idoine pour y faire face (cette intelligence étant si souvent langagière et les "atti degli uomini» si volontiers "actes de langage»).

Cette unité, indissociable de la question de l'auctorialité ${ }^{92}$, est d'ordre éthique (plus que strictement moral, ou alors au sens de la «morale provisoire» évoquée plus haut). Ce n'est pas seulement rendre acceptable la matière des nouvelles (plébéienne, licencieuse, etc.), c'est définir dans quelle mesure le réel sous toutes ses facettes peut acquérir droit de cité — ne plus avoir pour seule destinée que d'être toujours rejeté hors champ. Une fois ce droit (de tout évoquer et mettre en récit) acquis, le besoin de l'appareil «légitimant» de la structure enchâssée va peu à peu s'évanouir - car la disparition à terme de toute «cornice» (non péritextuelle),

90. Voici, ibid., p. 70-71, la suite de la citation de Jauss de la note précédente, qui reformule ce que Jolles disait du passage du Filocolo au Décaméron : "Quel est le plus grand plaisir de l'amoureux : penser à la femme aimée ou la voir?" [c'est la onzième des «questions d'amour" du Filocolo, IV 59] Des questions analogues et d'autres plus alambiquées se trouvent dans le partimen du Moyen Âge et y sont disputées comme dans une joute. [...] Selon l'hypothèse célèbre de A. Jolles, elles finirent par constituer la forme littéraire de la nouvelle dans laquelle un "événement inouï" met fin au débat. Mais le problème moral qui porte sur les normes impliquées dans le cas examiné n’est pas réglé ipso facto, si bien que le jeu de question et réponse peut se poursuivre et, comme dans le Décaméron de Boccace, la cas réglé en amène tout de suite un autre à sa suite.»

9I. C'est un point qui mériterait sans doute d'être développé, en direction de la peinture du Trecento, et en regardant de plus près les textes de Jolles sur les Primitifs. Voir, à ce propos, la suggestion de S. Contarini, " "Forme artistiche": Jolles e la teoria della novella", art. cité, p. II8-II9.

92. Voir M. Picone, Il Decameron come macrotesto: il problema della cornice, art. cité, p. 9-I3, où cette même question est abordée du point de vue sémiologique du dispositif narratologique complexe (opposé à la "collection») pensé et mis en œuvre. 
interroge ${ }^{93}$. Comme si la "cornice» de longue durée devenait dès lors un dispositif provisoire, contenait, arrivée à l'acmé de ses potentialités, la promesse de sa disparition, de son effacement futur — au profit du roman (mais la question est, on le sait, infiniment complexe) et du recueil simple de nouvelles, sans l'appareil d'une périnarrativité interne.

Jolles nous aide donc aussi à penser le dispositif formel du Décaméron comme un acte de pensée historiquement nécessaire - ou, mieux, comme un macro-acte de langage, produit dans le langage conçu comme travail; un langage qui est une pensée en acte et une pensée agissante, se confondant avec l'agir de l'écriture, qui "pragmatiquement» se précipite dans les images et la forme plutôt que dans les concepts et l'argumentation. Rien n'échappe dès lors à la juridiction artistique de la nouvelle. Ce qui permet ces intuitions? L'attention aux "formes", mais dans une conception dynamique de la forme : non seulement diachroniquement, dans la capacité d'une forme donnée de se transformer, mais aussi dans sa capacité de dialogue avec le monde comme avec d'autres formes. La capacité aussi d'engendrer du nouveau par un changement de l'ordre des choses qui se confond avec la faculté de fabriquer, qui se définit comme acte poétique.

93. Tout compte tenu du cas particulier du Cinquecento italien jusqu’à Bandello, tout boccacien; et, hors d'Italie, de la grande et célèbre exception de l'Heptaméron. Voir par exemple A. Asor Rosa, Decameron di Giovanni Boccaccio, art. cité, p. 559, ainsi que M. Cottino Jones, Il dir novellando: modello e deviazioni, Rome, Salerno, 1994, et R. Bragantini, Fra teoria e pubblico: la forma novellistica nel Cinquecento, dans La novella italiana, ouvr. cité, p. 445-467. 